\title{
C-C Bond Breaking in Addition-Elimination Reactions on Nitriles
}

\author{
Jean-Marc Mattalia*[a] and Paola Nava ${ }^{[a]}$
}

\begin{abstract}
The nucleophilic addition of organometallics to nitriles leads to an imine-type intermediate. When the $\alpha$-position is substituted with a carbanion stabilizing group, an elimination can follow resulting in a C-C bond breaking. This paper proposes a computational study of this step. Starting from the transnitrilation reaction by DMMN (dimethylmalononitrile), a comparison of several methods shows that the PBE0-D3 level is suitable for this study. The imine adducts display particularly stretched $\mathrm{C}-\mathrm{C}$ bond and thus the computed barriers are low. Both electronic and steric effects influence the leaving group ability as well as its interaction with the metal.
\end{abstract}

\section{Introduction}

Nitriles appear as a fundamental functional group in synthetic organic transformations ${ }^{[1]}$ and are present in many products of interest such as medicinally active natural products or pharmaceuticals. ${ }^{[2]}$ Therefore strategies for efficient cyanations have been considerably developed. ${ }^{[3]}$ One of them consists in using reagents that behave as formal $\mathrm{CN}^{+}$cation donors to achieve cyanation of a large variety of nucleophiles. ${ }^{[4]}$ The common feature in the proposed mechanisms is the formation of an addition intermediate followed by an elimination. Such fragmentation involves the breaking of various C-heteroatom bonds depending on the nature of the reagent. These include the $\mathrm{C}-\mathrm{N}$ (cyanamides) ${ }^{[5]} \mathrm{C}-\mathrm{O}$ (cyanates), ${ }^{[6]} \mathrm{C}-\mathrm{S}$ (thiocyanates) ${ }^{[7]}$ or $\mathrm{C}$ I (hypervalent iodine reagents) bonds. ${ }^{[8]}$

When the reaction takes place from simple nitriles, a C-C bond cleavage can occur as shown in Scheme 1. ${ }^{[9]}$ The nucleophilic addition to nitriles is a common way to synthesize carbonyl compounds after hydrolysis of the imine adduct intermediate (path a). ${ }^{[10]}$ These imine intermediates could also further react with nitriles leading to triazine derivatives. ${ }^{[11]}$ Here we shall concentrate only on the addition-elimination pathway, which is relevant when the $\alpha$-position is substituted with a carbanion stabilizing group. When the elimination proceeds (path b), the overall addition-elimination process corresponds to an electrophilic cyanation of the nucleophilic reagent. On the other hand, a reductive decyanation operates on the starting nitrile. This transformation allows for the removal of the cyano group after using its beneficial aspects in synthesis. ${ }^{[12]}$

[a] Dr. J.-M. Mattalia, Dr. P. Nava

Aix-Marseille Univ, CNRS, Centrale Marseille, iSm2, Marseille

France

E-mail: jean.marc-mattalia@univ-amu.fr

http://ism2.univ-amu.fr/fr/annuaire/ctom/ctom

Supporting information for this article is given via a link at the end of the document

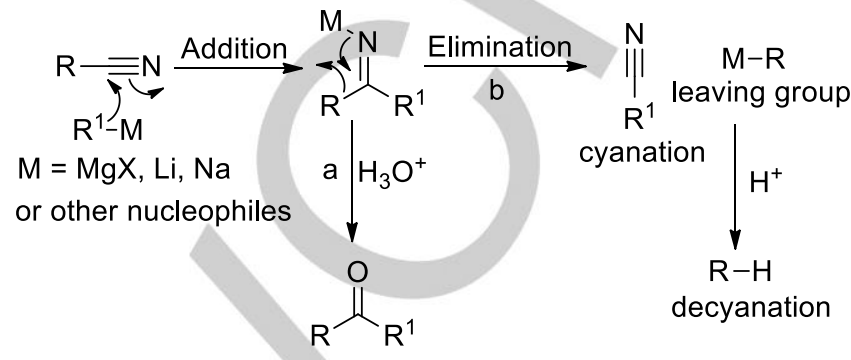

Scheme 1. General mechanism for cyanation and decyanation pathways.

The dual aspect of this reaction (cyanation and reductive decyanation) makes it very attractive. We have noticed that such addition-eliminations involving C-C bond breaking were proposed or could be involved in various chemical transformations. Scheme 2 is related to the reductive decyanation of aryl substituted tertiary nitriles with $\mathrm{NaH}$ in THF in the presence of Lil or Nal. ${ }^{[13]}$

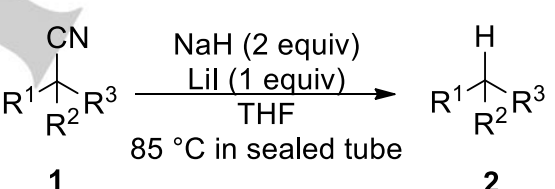<smiles>[R]c1ccc(C2CCCCC2)cc1</smiles>

2a $\mathrm{R}=\mathrm{Cl}, 81 \%, 2.5 \mathrm{~h}$

2b $\mathrm{R}=\mathrm{OMe}, 37 \%, 2.5 \mathrm{~h}$ $92 \%, 24 \mathrm{~h}$<smiles>c1ccc(C2CCCC2)cc1</smiles>

2c $84 \%, 2.5 \mathrm{~h}$<smiles>COc1ccc(C2(C=O)CCCCC2)cc1</smiles>

(along with $\mathbf{2 b}$ after $2.5 \mathrm{~h}$ and quenching with water)

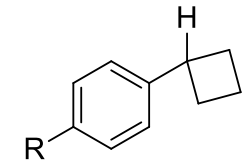

2d $\mathrm{R}=\mathrm{Ph}, 85 \%, 2.5 \mathrm{~h}$

2e $\mathrm{R}=\mathrm{OMe}, 70 \%, 32 \mathrm{~h}$
Scheme 2. Examples of decyanations using $\mathrm{NaH} / \mathrm{Lil}$ composite.

Experimental evidences and a computational study support the addition-elimination pathway described in Scheme 3 on dimethylphenylacetonitrile. The first step is the hydride addition to the $\mathrm{CN}$ triple bond with formation of an iminyl anion intermediate. The latter easily isomerizes to its isomer where a sodium cation$\pi$ interaction occurs. The last step involves a C-C bond cleavage and concerted proton transfer with elimination of $\mathrm{NaCN}$ and formation of the decyanation product. A similar mechanism has 
been previously proposed for the reductive decyanation promoted by $\mathrm{LiAlH}_{4}$ on 2,2-diphenylpropionitrile and related structures. ${ }^{[14]}$ Good yields are usually obtained after $2.5 \mathrm{~h}$. The 4methoxyphenyl group decreases the reaction rate (compare $\mathbf{2} \mathbf{b}, \mathbf{e}$ with $\mathbf{2 a}, \mathbf{c}, \mathbf{d}$ ). With the electron-rich aryl group, longer reaction times are needed and only a $37 \%$ yield of the decyanation product $\mathbf{2 b}$ is isolated after $2.5 \mathrm{~h}$. Simultaneously, a $42 \%$ yield of aldehyde 3 is formed after quenching with water, probably as a result of hydrolysis of the imine intermediate. ${ }^{[13]}$

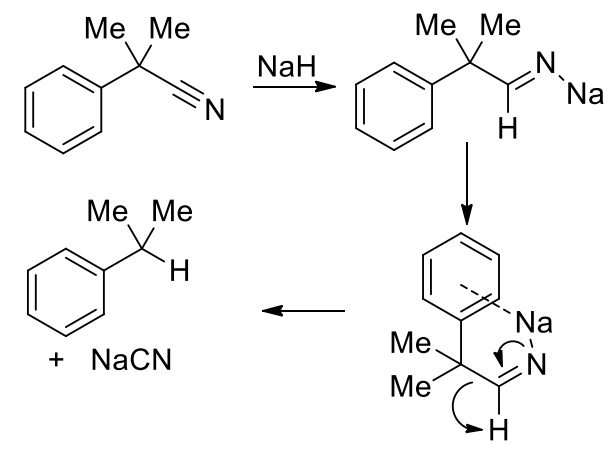

Scheme 3. Proposed pathway for decyanation by $\mathrm{NaH}$.

Kulp and Romanelli treated a series of alkyldiphenylacetonitriles with MeLi or PhLi in refluxing ether (Scheme 4). ${ }^{[15]}$ The authors did not investigate the mechanism but their findings are consistent with an addition-elimination pathway. The separation of products from the reaction mixture was difficult but the authors were able to isolate the decyanation product or, using $\mathrm{PhLi}$, benzophenone resulting from the addition of the excess of the aryllithium to the generated benzonitrile. The reactions with $\mathrm{PhMgBr}$ show that the reductive decyanation was more sluggish with Grignard reagents. The reactions of $\mathrm{PhMgBr}$ with 2,2-diphenylbutanenitrile or 2,2diphenylpropanenitrile lead exclusively to the addition product. From 2,2-diphenylhexanenitrile, a $72 \%$ yield of addition was obtained in refluxing toluene but, interestingly, $80 \%$ of decyanation was observed around $180^{\circ} \mathrm{C}$ in an experiment where "the solvent was inadvertently evaporated". ${ }^{[15]}$ The decyanation of diphenylacetonitriles with Grignard reagents was previously described but reports suggest that the amount of decyanation might strongly depend on nitrile structures and experimental conditions. ${ }^{[16]}$

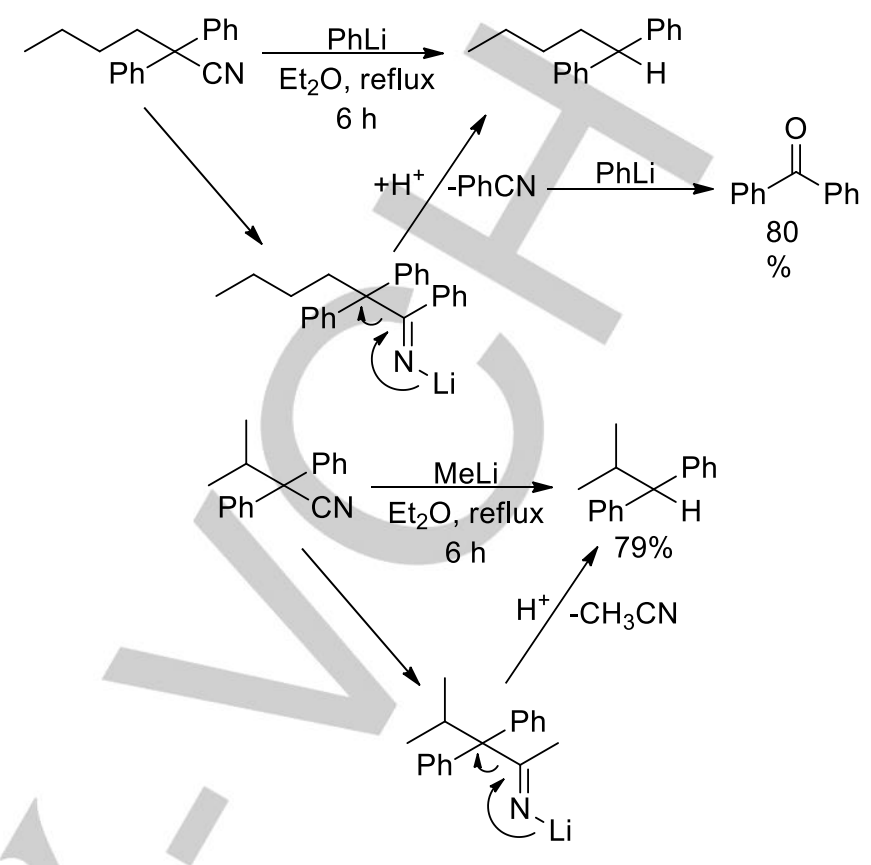

Scheme 4. Reductive decyanation promoted by organolithiums.

We propose, through a computational study, to bring a fresh perspective to this reaction. In a first part, based on the transnitrilation of organometallics using dimethylmalononitrile $(\mathrm{DMMN}),{ }^{[17]}$ we compare calculations that lead us to select the more appropriate computational level. Then, by changing the structure of the nitrile and nucleophile, we investigate the structure-reactivity relationship of the imine-type intermediate. As much as possible our discussion will be related to experimental data. Newly, our study highlights some intermediates with stretched $\mathrm{C}-\mathrm{C}$ bonds. The generation and characterization of species with unusually long $\mathrm{C}-\mathrm{C}$ bonds has received lately great interest from the point of view of the computational description as demonstration of the importance of intramolecular London dispersion interactions. ${ }^{[18]}$

Our preliminary study on the computational approach has been motivated by the work by Reeves et al. The authors investigated the transnitrilation of aryl Grignard reagents with dimethylmalononitrile (Scheme 5) to obtain the benzonitrile and the isobutyronitrile anion. ${ }^{[19]}$ Aryllithiums generated in situ at $78{ }^{\circ} \mathrm{C}$ from aryl bromides and $n \mathrm{BuLi}$ also react with DMMN at $78^{\circ} \mathrm{C}$-r.t. to yield aryl nitriles in high yields. Interestingly, they further developed a transnitrilation/ $S_{N} A r$ sequence where the isobutyronitrile anion was used as nucleophile in an aromatic substitution reaction. ${ }^{[20]}$

For this transnitrilation reaction, a mechanism through an imine adduct was proposed. Experimental evidences suggest an equilibrium between the imine and the dissociated complex: IR monitoring was consistent with the formation of benzonitrile, the isobutyronitrile anion and the imine intermediate. Moreover, quenching the reaction mixture after $30 \mathrm{~min}$ with water, led to the corresponding ketone in $15 \%$ isolated yield. Small quantities of the ketone were detected "even after extended reaction times". ${ }^{[19]}$ 


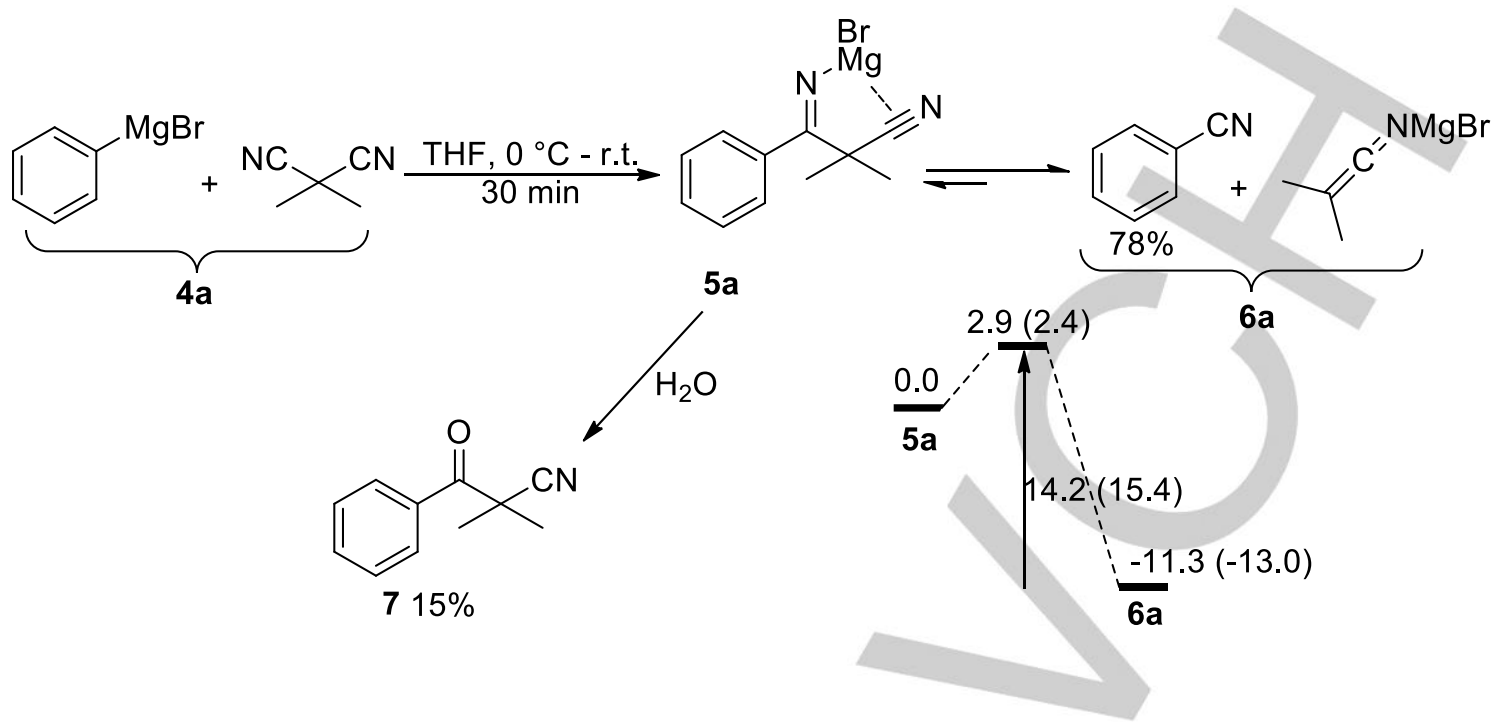

Scheme 5. Transnitrilation of Grignard reagents with dimethylmalononitrile. Evidence for the ketimine intermediate. The energetic diagram as obtained in reference 19 is reproduced: ZPE-corrected electronic energies (in $\mathrm{kcal} \mathrm{mol}^{-1}$ ) were at the B3LYP/LanL2DZ level of theory, by using the SMD implicit solvation model and one explicit THF molecule. Gibbs-free-energy values are shown in brackets.

Their computational investigation indicates, however, a clear exergonic retro-Thorpe fragmentation from the imine intermediate $\mathbf{5 a}$, the fragmented complex $\mathbf{6 a}$ is $13 \mathrm{kcal} \mathrm{mol}^{-1}$ lower than the proposed intermediate. Furthermore, the forward process has a tiny barrier of $2.4 \mathrm{kcal} \mathrm{mol}^{-1}$ and, as a consequence of the exergonicity, a much higher reverse barrier of $15.4 \mathrm{kcal} \mathrm{mol}^{-1}$. Even if the equilibrium should be displaced towards the fragmented complex, favored from an entropic point of view, our question is whether the computed difference in energy between the imine $\mathbf{5 a}$ and the fragmented complex $\mathbf{6 a}$ is somewhat overestimated.

\section{Results and Discussion}

The transnitrilation of organometallics dimethylmalononitrile: a preliminary study

We reexamine first results obtained from Reeves et al. on the fragmentation problem. We computed the whole reaction pathway including the addition step of complex $4 a^{\left[{ }^{[21]}\right.}$ Scheme 6 outlines a comparison between PBE0-D3 and B3LYP/def2TZVP levels of theory. In these calculations one explicit THF molecule was included. Our B3LYP values differ only slightly from those from Reeves et al., by at most $2.7 \mathrm{kcal} \mathrm{mol}^{-1}$ in the energies of $6 \mathbf{a}$ and

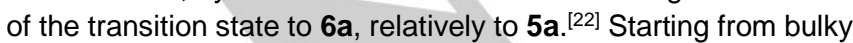
reactants, additions of Grignard reagents to nitriles are slow processes. ${ }^{[23]}$ However, hindered DMMN appears quite reactive as a result of an increases of electrophilicity of the cyano group. The computed barriers for the addition is quite high at the B3LYP level, values at the PBE0-D3 level fit better with the feasibility of the reaction at experimental conditions $\left(30\right.$ minutes, $0^{\circ} \mathrm{C}-$ r.t. $)$.
Besides the energy of $\mathbf{6 a}$, a remarkable difference between the computed values is the larger stability of intermediate $\mathbf{5 a}$ with the PBE0-D3 method: the fragmentation barrier increases while the reaction appears much more exothermic with the B3LYP method than with PBE0-D3. As expected, the entropic term favors the fragmentation and the dissociated complex $6 \mathbf{a}$ is stabilized in term of $\Delta \mathrm{G}$.

In order to compare the accuracy of several methods, we focused on electronic energies (containing the ZPE). From here, reported energies are relative to the imines. Initially, we performed calculations on very simple model systems, where the $\mathrm{MgBr}$ moiety is replaced by a hydrogen also bearing a partial positive charge. We are aware that this reaction would not occur, ${ }^{[24]}$ but it allows for systematic accurate calculations to detect a possible B3LYP over-stabilization of the fragmented complex with respect to the imine. A set of several starting imine intermediates was chosen, to be able to quantify possible effects due to the nature of groups $R^{1}$ and $R^{2}$. We compare results from B3LYP, ${ }^{[25]}$ PBE0, ${ }^{[25 c, 25 e, 26]}$ PBE0-D3, ${ }^{[27]}$ and M06-2X ${ }^{[28]}$ to reference SCS$\mathrm{MP}^{[29]}$ and $\operatorname{CCSD}\left(\mathrm{F} 12^{*}\right)(\mathrm{T})^{[30]}$ data (Figure 1). Calculations for the $\mathrm{MgBr}$ containing systems were performed by using the DFT and the SCS-MP2 methods. Results are collected in Figure 2. Structures have been optimized at each level, but for the $\operatorname{CCSD}\left(\mathrm{F} 12^{*}\right)(\mathrm{T})$ level, results are obtained from single-point calculations on PBE0-D3 structures. ${ }^{[30]}$ The basis set if of def2TZVP quality ${ }^{[31]}$ for DFT and SCS-MP2 calculations. We used the cc-pVDZ-F12 ${ }^{[32]}$ with the appropriate auxiliary basis sets for the $\operatorname{CCSD}\left(\mathrm{F} 12^{*}\right)(\mathrm{T})$ calculations. The RI-J technique was used to reduce the computational cost. ${ }^{[31 \mathrm{a}]}$ 


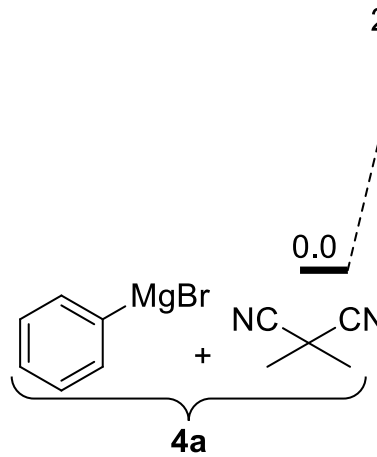

$20.4(24.3)$

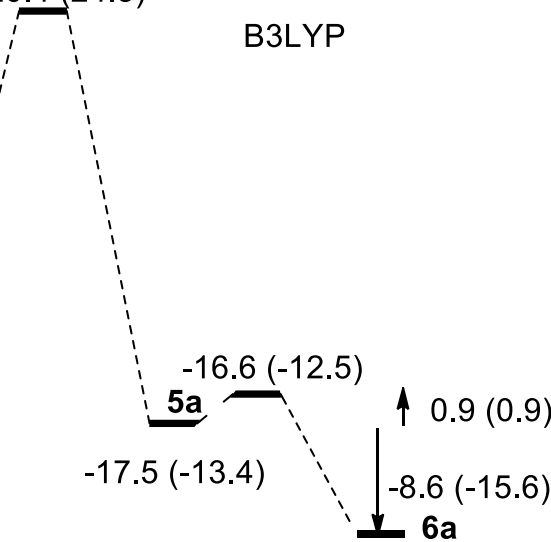

$-26.1(-29.0)$

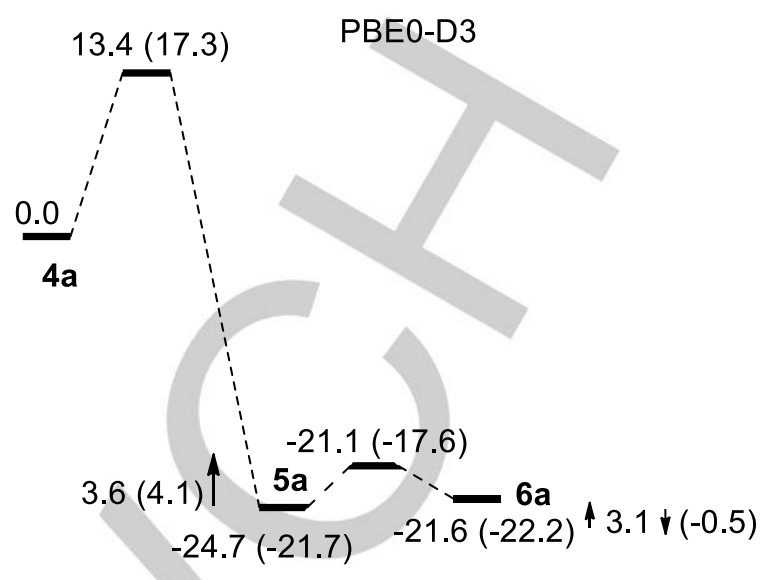

Scheme 6. Comparison between computed energetic profiles for the transnitrilation of pheylmagnesium bromide with DMMN. ZPE-corrected electronic energies (in $\mathrm{kcal} \mathrm{mol}^{-1}$ ) are obtained at the B3LYP/def2-TZVP and PBE0-D3/def2TZVP levels of theory. Gibbs-free-energy values at $298.15 \mathrm{~K}$ are shown in brackets. One molecule of explicit THF was added.

In the model systems, the elimination processes are endothermic. The SCS-MP2 results are very close to those obtained with the reference level. The B3LYP results for the difference in energy between imines and fragmented complexes deviate from the $\operatorname{CCSD}\left(\mathrm{F} 12^{*}\right)(\mathrm{T})$ reference results by 5 up to $16 \mathrm{kcal} \mathrm{mol}^{-1}$. The other functionals are performing much better, notably PBE0-D3 and M06-2X have errors of less than $5 \mathrm{kcal} \mathrm{mol}^{-1}$. It is noteworthy that B3LYP and, to a lesser extent, PBE0 show the larger errors for the most substituted cases $\left(R^{1}, R^{2}\right)=(\mathrm{Me}, \mathrm{Me})$ and $(\mathrm{Ph}, \mathrm{Me})$ : the lack of dispersion corrections for those functionals induces an overestimation of the steric decompression that follows the elimination process, leading to an artificial destabilization of the imine. Values for computed barriers (from imines to fragmented complexes) are not surprising: as expected, DFT methods are all underestimating the energies of the transition states (see Figure S1 in Supporting Information).

In order to validate the results obtained for the model systems, $\mathrm{MgBr}$ containing systems were treated by several methods. We could not perform $\operatorname{CCSD}\left(\mathrm{F} 12^{*}\right)(\mathrm{T})$ calculations on them, however the $(\mathrm{Ph}, \mathrm{Me})$ case was computed at the SCS-MP2 level, as this method gives very close results to the $\operatorname{CCSD}\left(F 12^{*}\right)(T)$. Energies and barriers are drastically reduced with respect to the model systems (Figure 2). Trends on the methods are as for the model systems. It is only with the B3LYP functional that the fragmented complex is clearly energetically favored. This is remarkable for the $(\mathrm{Ph}, \mathrm{Me})$ case, where the B3LYP result suffers of the overestimation of the structural decompression. With the PBE0 method, the product is almost isoenergetic with respect to the imine and becomes, using dispersion correction, slightly disfavored as with the other methods. However, we remind the reader that Figure 2 shows differences in total energies (corrected by the ZPE) but a stabilization of the complex is expected by considering the entropic term.

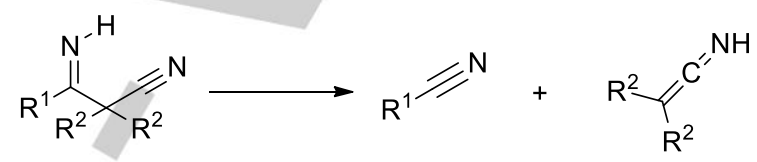

$(\mathrm{H}, \mathrm{H})$

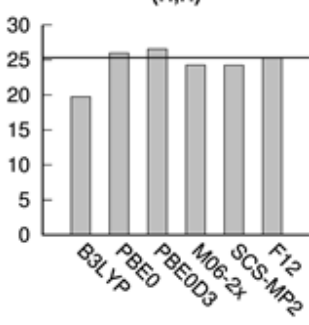

$(\mathrm{H}, \mathrm{Me})$

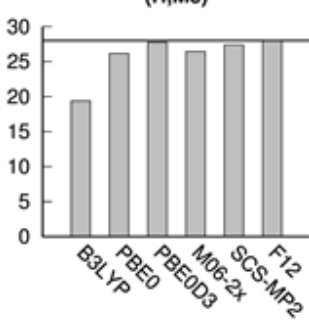

$(\mathrm{Ph}, \mathrm{H})$

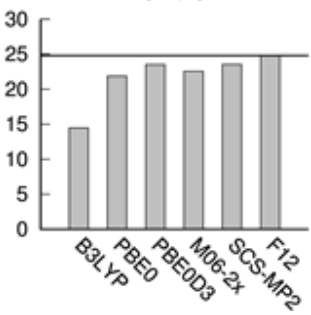

$(\mathrm{Me}, \mathrm{H})$

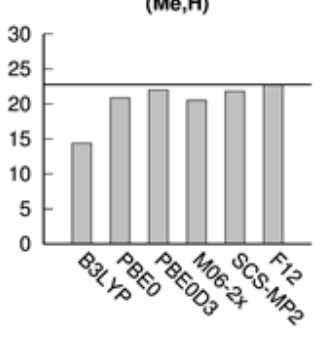

(Me,Me)

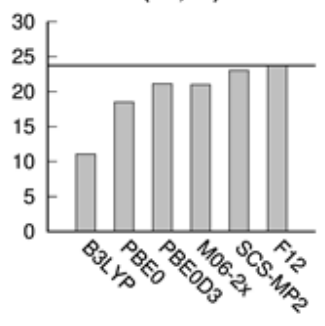

$(\mathrm{Ph}, \mathrm{Me})$

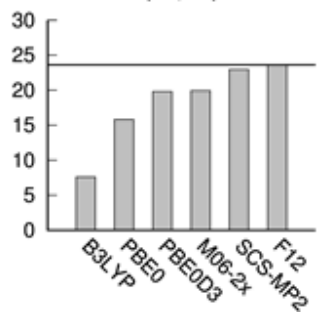

Figure 1. ZPE-corrected electronic energies in $\mathrm{kcal} \mathrm{mol}^{-1}$ of the fragmented complex with respect to the imine, for the model systems. F12 stays for $\operatorname{CCSD}\left(F 12^{*}\right)(T)$ calculations. Substituents in brackets refer to $\left(R^{1}, R^{2}\right)$. Straight lines indicate $\operatorname{CCSD}\left(\mathrm{F} 12^{*}\right)(\mathrm{T})$ reference values. 


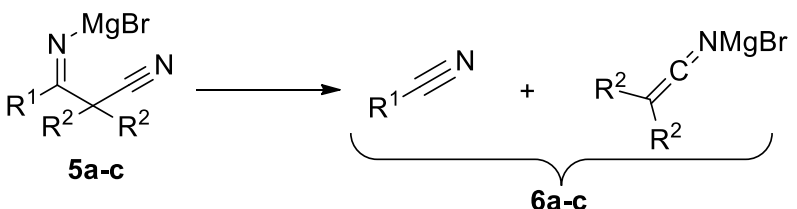

(Me,Me) Ts

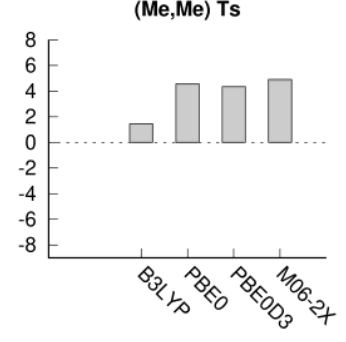

$(\mathrm{Ph}, \mathrm{H}) \mathrm{Ts}$

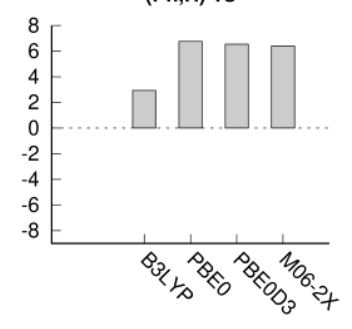

$(\mathrm{Ph}, \mathrm{Me}) \mathrm{Ts}$
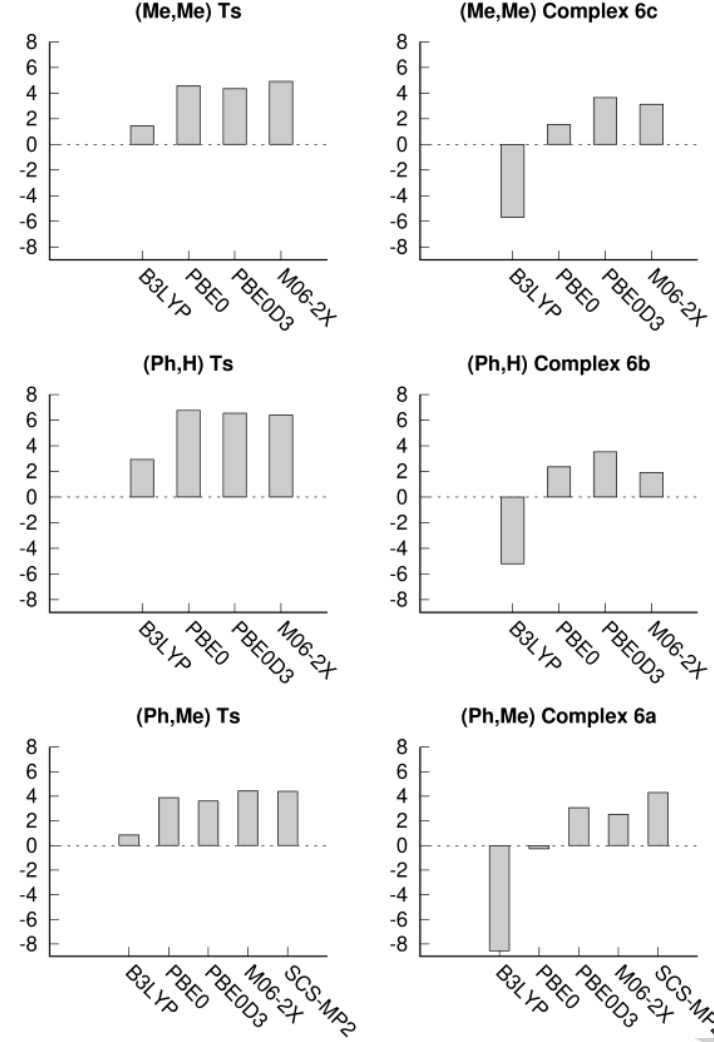

(Ph,H) Complex 6b

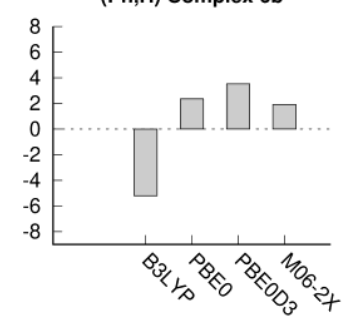

(Ph,Me) Complex 6a

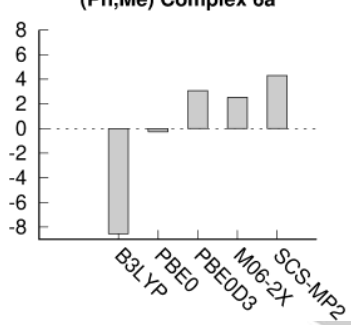

Figure 2. ZPE-corrected electronic energies in $\mathrm{kcal} \mathrm{mol}^{-1}$ of the transition state (Ts) and fragmented complex with respect to the imine, for the $\mathrm{MgBr}$ containing system. Substituents in brackets refer to $\left(R^{1}, R^{2}\right)$. One molecule of explicit THF was added.

Is there an explanation for the behavior of the B3LYP functional that destabilizes 5a with respect to $6 a$ ? Why does the $\mathrm{MgBr}$ mediated elimination, which implies the breaking of a C-C bond, have such low barriers? To give some insights, the computed $\mathrm{C} 1$ $\mathrm{C} 2$ bond distances in the imines are reported in Table 1. The B3LYP values are systematically somewhat longer than those computed at the other levels: in the model systems the discrepancy is very small (about $1 \mathrm{pm}$ with respect to the other methods), but it becomes more relevant when $X=\operatorname{MgBr}$ (3 to 6 $\mathrm{pm})$. This overestimation of the $\mathrm{C}-\mathrm{C}$ bond distance correlates well with the energetic results, since it implies that the imine systems are too destabilized with respect to the transition states and the fragmented complexes at the B3LYP level. Our calculations reveal a difficulty for the B3LYP method to properly describe a systems containing a stretched C-C bond (in this case $\mathrm{Csp}^{2}-\mathrm{Csp}^{3}$ ). We have already encountered this problem for (benzo)cyclobutene derivatives with strained C-C bonds, for which differences in energy between closed and open forms (dienes) are also biased with the B3LYP functional in favor of the open forms. ${ }^{[33]}$

Table 1. Computed C1-C2 distances in the imine systems (pm). Substituents in brackets refer to $\left(R^{1}, R^{2}\right)$.<smiles>[Y]N=C([R])C([R])([R])C#N</smiles>

\begin{tabular}{|c|c|c|c|c|c|}
\hline & B3LYP & PBE0 & PBE0-D3 & M06-2X & SCS-MP2 \\
\hline \multicolumn{6}{|c|}{ MODEL SYSTEMS $(X=H)$} \\
\hline$(\mathrm{Me}, \mathrm{Me})$ & 155.4 & 154.2 & 154.2 & 154.2 & 154.1 \\
\hline$(\mathrm{Me}, \mathrm{H})$ & 153.6 & 152.6 & 152.6 & 152.9 & 152.9 \\
\hline$(\mathrm{H}, \mathrm{H})$ & 152.4 & 151.5 & 151.6 & 151.9 & 152.1 \\
\hline$(\mathrm{H}, \mathrm{Me})$ & 153.4 & 152.4 & 152.4 & 152.6 & 152.5 \\
\hline$(\mathrm{Ph}, \mathrm{Me})$ & 155.8 & 154.5 & 154.4 & 154.4 & 154.3 \\
\hline$(\mathrm{Ph}, \mathrm{H})$ & 153.7 & 152.6 & 152.6 & 152.9 & 153.0 \\
\hline \multicolumn{6}{|c|}{$\mathrm{X}=\mathrm{MgBr}$} \\
\hline$(\mathrm{Me}, \mathrm{Me})$ & 166.2 & 162.1 & 161.9 & 161.8 & -- \\
\hline$(\mathrm{Ph}, \mathrm{Me})$ & 166.7 & 162.4 & 162.4 & 161.8 & 160.8 \\
\hline$(\mathrm{Ph}, \mathrm{H})$ & 163.6 & 160.4 & 160.5 & 160.6 & -- \\
\hline
\end{tabular}

We notice, however, that the peculiar feature of the imine is indeed the stretched C-C bond (Table 1). The $\mathrm{C}-\mathrm{C}$ bond distances are at the upper limit of usual single $\mathrm{C}-\mathrm{C}$ bonds $\left(\mathrm{Csp}^{2}-\mathrm{Csp}^{3}=151\right.$ $\mathrm{pm})$ already in the model systems. The feature is strongly enhanced by the $\mathrm{MgBr}$ group, where $\mathrm{C}-\mathrm{C}$ bond distances are computed to be about $160 \mathrm{pm}$, clearly longer than usual single $\mathrm{C}$ $\mathrm{C}$ bonds. This structural feature is significant, since it reveals that the $\mathrm{C}-\mathrm{C}$ bond is weakened in the imine and explains that barriers for elimination are low.

The lack of dispersion in the B3LYP and PBE0 treatments leads to an overestimation of the steric decompression that follows the elimination. This could explain, at least in part, the reason why the barrier from $\mathbf{4 a}$ to $\mathbf{5 a}$ is higher at the B3LYP than at the PBE0-D3 level (Scheme 6). Moreover, the B3LYP functional does not treat properly the imine systems, resulting in too high an energy for stretched $\mathrm{C} 1-\mathrm{C} 2$ with respect to the fragmented complex. The other methods better account for the equilibrium between $5 \mathrm{a}$ and $6 \mathbf{a}$ and the use of dispersion improves PBE0 results. In recent contributions, ${ }^{[18]}$ the existence of species with long Csp ${ }^{3}-\mathrm{Csp}^{3}$ bonds has been demonstrated and successfully treated by using DFT methods including corrections for dispersion effects. For those cases, the existence of the $\mathrm{C}-\mathrm{C}$ bond, which is elongated, is related to strong intramolecular London interactions between bulky groups and the B3LYP-D3(BJ) level of computation recovers a good description of those systems (as M06-2X or PBE0-D3). In our case, it is necessary to take into account 
dispersion effects as well, however the elongation of the $\mathrm{C}-\mathrm{C}$ bond is inherent to the nature of the imine and is relevant for cases where the steric hindrance is modest (Table 1).

Finally, the feasibility of the elimination is related to the weakness of the $\mathrm{C} 1-\mathrm{C} 2$ bond. We shall check this correlation in the following section for several cases, which will be treated at the PBE0-D3 level of theory.

\section{Comparison of study cases.}

Table 2 collects results for structures related to Schemes 2-5. All structures displayed in Table 2 show stretched $\mathrm{C} 1-\mathrm{C} 2$ bonds (158-164 pm). Differences in distances are small but, overall, for related structures, the shorter values lead to higher barriers. This is obvious when including explicit solvation in $\mathbf{9 b}$, or when $\mathrm{Li}$ is replaced with $\mathrm{MgBr}$ (compare 9c and 10).

We discuss first effects of the leaving group $M-R$ (Scheme $1, M=$ $\mathrm{MgBr}$, Na, Li). We compare eliminations from adducts of dimethylmalononitrile (structures $\mathbf{5 a}-\mathbf{5 b}, \mathbf{M}=\mathrm{MgBr}$ ). When the two methyl groups are replaced with hydrogens, the $+l$ effect of the methyl groups is expected to disfavor elimination by destabilizing the incipient negative charge at the $\alpha$-position. ${ }^{[34]}$ Nevertheless, the barrier increases and the elimination appears more endothermic: the relief of steric strain upon elimination is more pronounced and overcomes the unfavorable electronic effect.

We consider $\alpha$-aryl substituted imines, $M=\mathrm{Na}$ : structures $\mathbf{8 a - 8 b}$ show that the substitution of the phenyl group with a $p$-methoxy leads to a significant increase of the elimination barrier in agreement with the decrease of the reaction rate experimentally observed (Scheme 2). Compared to the phenyl, the $p$ methoxyphenyl group destabilizes the negative charge triggered at the benzylic position upon the $\mathrm{C}-\mathrm{C}$ bond cleavage, steric effects are negligible and the barriers fit with the $\mathrm{p} K_{\mathrm{a}}$ of the leaving group. By comparing structures $9 a$ and $9 b(M=L i)$, we notice that replacing a methyl group with a phenyl at the $\alpha$-position brings beneficial electronic and steric effects and makes the $\mathrm{C}-\mathrm{C}$ bond cleavage easier. ${ }^{[35]}$ When the methyl group in $\mathbf{9 b}$ is replaced with a phenyl group (9c) the barrier increases. In this case, the electron-withdrawing effect of the phenyl group could play a role by stabilizing the imine. ${ }^{[6 e, 36]}$ Finally, leaving groups affect both electronically and sterically the reactivity: in the transition state, delocalization of the negative charge at the $\alpha$-position and steric decompression are favorable factors.

We discuss now $\mathrm{Li}$ and $\mathrm{MgBr}$ by comparing structures $9 \mathrm{c}$ with $\mathbf{1 0}$, and 5a with 11. As stated in the introduction for alkyldiphenylacetonitriles, elimination of Grignard intermediates appears more sluggish than lithium counterparts. The higher reactivity of organolithiums compared with Grignard reagents has been related to the larger electronegativity of $\mathrm{Mg}$ (1.31) compared to $\mathrm{Li}(0.98):{ }^{[37]}$ in the elimination reaction $\mathrm{MgBr}$ could retain the electron pair of the N-Metal bond more than Li. As expected, when $\mathrm{Li}$ is replaced with $\mathrm{MgBr}$ on $\alpha$-diphenyl substituted imines, the barrier strongly increases and the reaction becomes endothermic (9c and 10). Moreover, the transition states require a metal assistance in the form of a cation- $\pi$ interaction; the $\mathrm{Li}-\mathrm{C}$ (aromatic) distances average is $238 \mathrm{pm}$ in the transition state starting from imine 9c. This interaction is already present in $9 \mathrm{c}$ where the $\mathrm{Li}$ $\mathrm{C}$ (aromatic) distances vary from 236 to 267 pm (average 252 pm,
Figure 3). ${ }^{[38]}$ In the $\mathrm{MgBr}$ case, the $\mathrm{Mg}-\mathrm{C}$ (aromatic) distances in the imine 10 vary from 259 to 328 pm (average $294 \mathrm{pm}$ ) and are far from values required in the transition state (average $259 \mathrm{pm}$, Figure 4).

Table 2. Comparison between various imines for the elimination reaction. ZPE-corrected electronic energies (in $\mathrm{kcal} \mathrm{mol}^{-1}$ ) are obtained at the PBE0D3/def2TZVP level of theory. Gibbs-free-energy values at $298.15 \mathrm{~K}$ are shown in brackets.

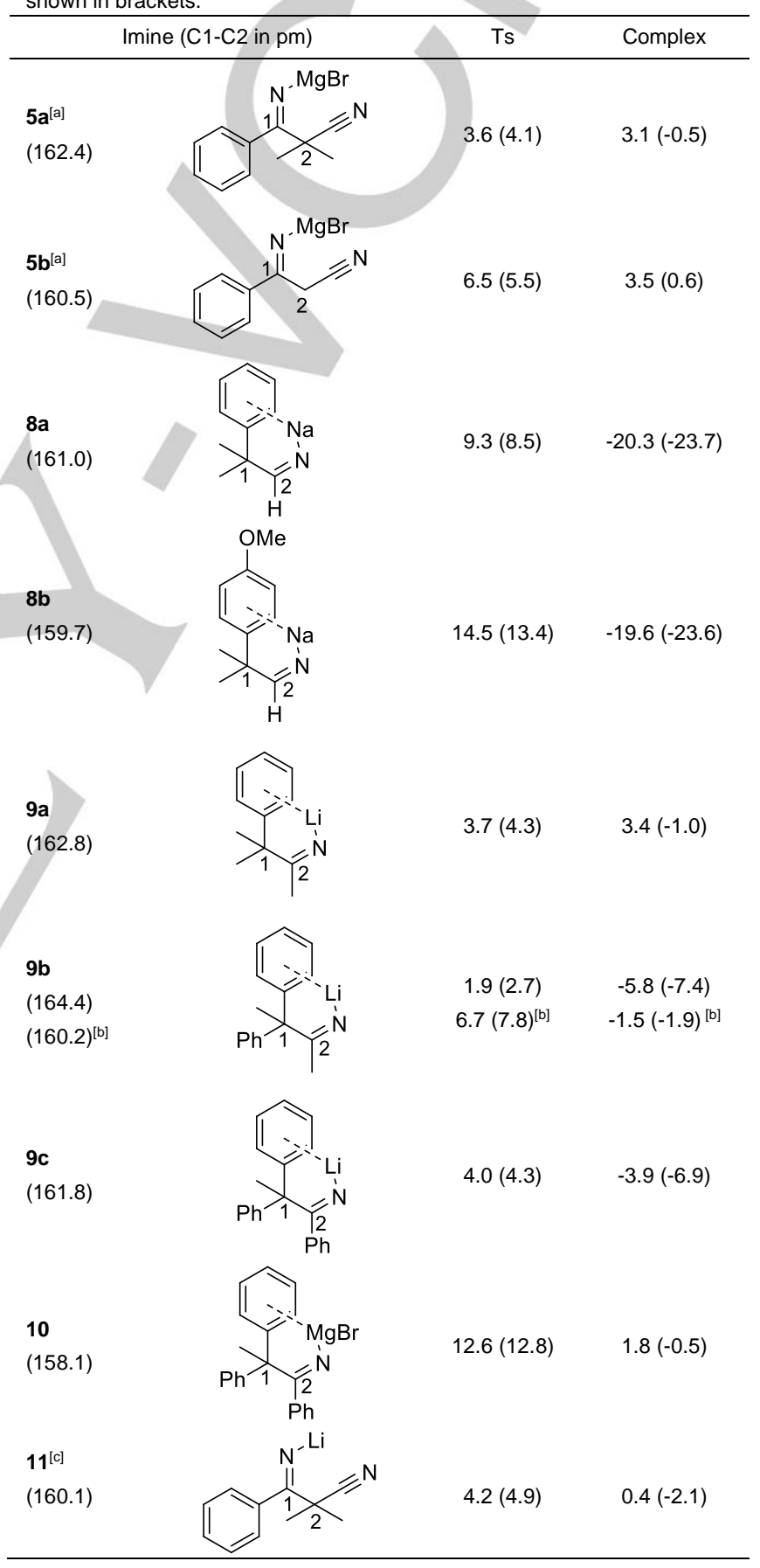

[a] 1 explicit molecule of THF. [b] 2 explicit molecules of $\mathrm{Me}_{2} \mathrm{O}$. [c] 2 explicit molecules of THF. 


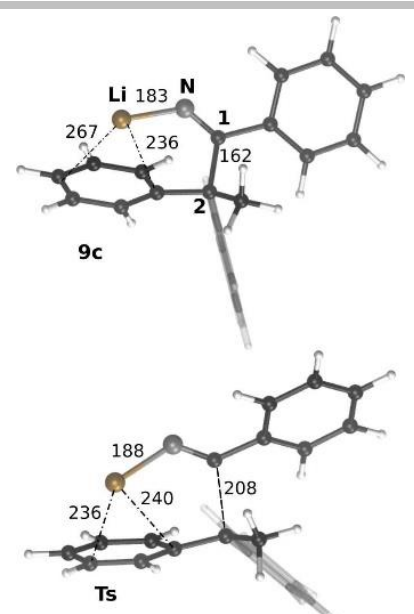

Figure 3. Imine 9c and corresponding transition state. Distances are in pm.

In contrast, for imines $\mathbf{1 1}$ and $\mathbf{5 a}$ barriers are similar and the higher reactivity in the Li case is no longer observed, even if the reaction is slightly more exergonic for the Li-complex.

In accordance, transnitrilations from dimethylmalononitrile require similar reaction conditions regardless the nature of the organometallic reagent. The Metal-N(nitrile) distances are similar for $\mathrm{MgBr}$ and $\mathrm{Li}$ in starting imines 5a and 11 (about $227 \mathrm{pm}$ ) and the corresponding transition states (about $208 \mathrm{pm}$ ). The more electronegative $\mathrm{MgBr}$ group could help to "pull" more efficiently the electron pair of the breaking $\mathrm{C}-\mathrm{C}$ bond and then increase the leaving group ability. This effect could overcome the unfavorable effect of $\mathrm{MgBr}$ regarding the electron pair at the imine group. We mention that for structure $\mathbf{1 1}$ two explicit THF molecules were included in the calculations to preserve a tetracoordination for the lithium as for the magnesium counterpart.

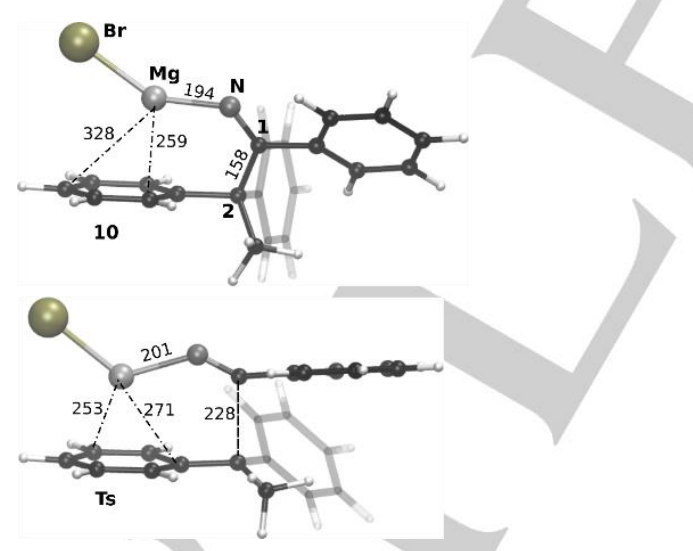

Figure 4. Imine $\mathbf{1 0}$ and corresponding transition state. Distances are in pm.

In this discussion, we considered explicit solvation only for adducts of dimethylmalononitrile. If we add two explicit molecules of dimethylether to the lithium intermediate $\mathbf{9 b}$, the barrier drastically increases. The cation- $\pi$ interaction strongly decreases with the solvation of the $\mathrm{Li}$ cation; ${ }^{[39]}$ the $\mathrm{Li}-\mathrm{C}$ (aromatic) distances vary from 270 to $395 \mathrm{pm}$ (compared with 239 to $262 \mathrm{pm}$ without $\mathrm{Me}_{2} \mathrm{O}$ ) and the average distance is $336 \mathrm{pm}$ (251 pm without $\mathrm{Me}_{2} \mathrm{O}$ ). These distances are higher than values reported for related $\pi$-complexes. ${ }^{[38]}$ This effect points out the importance of the metal assistance in the leaving group ability: a weak metal- $\pi$ interaction decreases the electron-withdrawing ability of the phenyl group.

Explicit solvent increases slightly the elimination barrier; however these addition-elimination reactions are often performed in Lewis basic solvents (Schemes 2-5). The use of Lewis basic solvent has a strong influence on the aggregation state particularly of the organolithium species. Even if reactions of dimers or other aggregate states have been described, deaggregation by Lewis bases usually lead to an increase of reactivity and a large number of studies lead to the conclusion that monomers are reactive species. ${ }^{[40]}$ Furthermore, at the elimination stage, one can envision that an equilibrium is possible, even if not favorable, ${ }^{383 a}$ ${ }^{41]}$ where a solvent molecule on the metal could be displaced by the phenyl group to generate a cation $\pi$ - interaction favoring the C-C bond breaking.

\section{Conclusion}

Transnitrilation and reductive decyanation reactions proceed through an addition-elimination pathway. The nucleophilic addition leads to an imine intermediate displaying a clearly stretched C-C bond. This implies that barriers are easy to overcome. The overall process is favored from an entropic point of view. Steric effects, $p K_{\mathrm{a}}$ of the acid related to the leaving group as well as the nature of the initial nucleophile influence barriers and energies.

From a computational point of view, the B3LYP functional is not appropriate for the description of the elimination and the PBE0D3 method was used, instead. The dispersion correction helps for a correct estimation of the steric decompression effect, otherwise overestimated.

As previously proposed, the mechanism of addition-elimination could be an alternative to the classical hydrolysis-decarboxylation pathway for the reductive decyanation of diphenylacetonitriles in basic medium. ${ }^{[42]}$ This pathway could be significant for the reaction of organolithiums with 1-cyanotetrahydronaphthalene derivatives (instead of the pathway through a four center transition state $)^{[43]}$ or the reaction of Grignard reagents with triarylacetonitriles (instead of an electron transfer mechanism). ${ }^{[44]}$ Finally, even if the potassium case was not considered in our work, the investigation of the addition-elimination mechanism may bring a fresh perspective to KHMDS mediated $S_{N} A r$-decyanation reactions starting from dibenzothiophene dioxides and

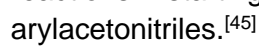

\section{Acknowledgments}

We thank Mr. Alexander Punter for language revision.

Keywords: density functional theory calculations ; elimination ; imine adduct ; transnitrilation ; reactive $\mathrm{C}-\mathrm{C}$ bond 
[1] a) F. F. Fleming, Z. Zhang, Tetrahedron 2005, 61, 747-789; b) N. Otto, T. Opatz, Chem. Eur. J. 2014, 20, 13064-13077; c) B. R. Pitta, O. W. Steward, F. F. Fleming, J. Org. Chem. 2018, 83, 2753-2762; d) X. Yang, F. F. Fleming, Acc. Chem. Res. 2017, 50, 2556-2568.

[2] a) F. F. Fleming, Nat. Prod. Rep. 1999, 16, 597-606; b) F. F. Fleming, L. Yao, P. C. Ravikumar, L. Funk, B. C. Shook, J. Med. Chem. 2010, 53 7902-7917.

[3] a) J. Kim, H. J. Kim, S. Chang, Angew. Chem., Int. Ed. 2012, 51, 1194811959; b) J.-L. Liu, Z.-F. Zhu, F. Liu, Org. Lett. 2018, 20, 720-723; c) P. Merino, in Comprehensive Organic Synthesis, Vol. 1 (Eds.: P. Knochel, G. A. Molander), Elsevier, 2014, pp. 697-750.

[4] J. Schörgenhumer, M. Waser, Org. Chem. Front. 2016, 3, 1535-1540.

[5] a) M. Chaitanya, P. Anbarasan, Org. Biomol. Chem. 2018, 16, 70847103; b) V. N. Murthy, S. P. Nikumbh, S. P. Kumar, L. V. Rao, A. Raghunadh, Tetrahedron Lett. 2015, 56, 5767-5770; c) K. Sudhakar, E. Y. Goud, G. Thirupathi, Y. Hemasri, K. Mahesh, A. Raghavendar, H. S. Babu, K. S. Kumar, ChemistrySelect 2018, 3, 1176-1179; d) P. Anbarasan, H. Neumann, M. Beller, Chem. Eur. J. 2010, 16, 4725-4728; e) P. Anbarasan, H. Neumann, M. Beller, Chem. Eur. J. 2011, 17, 42174222; f) T. V. Hughes, M. P. Cava, J. Org. Chem. 1999, 64, 313-315; g) M. Kasthuri, H. S. Babu, K. S. Kumar, C. Sudhakar, P. V. N. Kumar, Synlett 2015, 26, 897-900; h) K. Kiyokawa, T. Nagata, S. Minakata, Angew. Chem., Int. Ed. 2016, 55, 10458-10462; i) O. Loreau, N. Camus, F. Taran, D. Audisio, Synlett 2016, 27, 1798-1802; j) Y.-q. Wu, D. C. Limburg, D. E. Wilkinson, G. S. Hamilton, Org. Lett. 2000, 2, 795-797; k) Y. Yang, Y. Zhang, J. Wang, Org Lett 2011, 13, 5608-5611; I) R. Crossley, R. G. Shepherd, J. Chem. Soc., Perkin Trans. 1 1985, 2479-2481.

[6] a) J. S. Koo, J. I. Lee, Synth. Commun. 1996, 26, 3709-3713; b) D. Martin S. Rackow, Chem. Ber. 1965, 98, 3662-3671; c) R. E. Murray, G. Zweifel, Synthesis 1980, 150-151; d) N. Sato, Q. Yue, Tetrahedron 2003, 59, 5831-5836; e) N. Thoai, M. Rubinstein, C. Wakselman, J. Fluorine Chem. 1982, 20, 271-276

[7] a) R. Akula, Y. Xiong, H. Ibrahim, RSC Adv. 2013, 3, 10731-10735; b) D. Kahne, D. B. Collum, Tetrahedron Lett. 1981, 22, 5011-5014; c) I. Klement, K. Lennick, C. E. Tucker, P. Knochel, Tetrahedron Lett. 1993, 34, 4623-4626; d) A. Miyashita, I. Nagasaki, A. Kawano, Y. Suzuki, K.-i. Iwamoto, T. Higashino, Heterocycles 1997, 45, 745-755; e) I. Nagasaki, Y. Suzuki, K.-i. Iwamoto, T. Higashino, A. Miyashita, Heterocycles 1997 , 46, 443-450; f) A. M. Van Leusen, J. C. Jagt, Tetrahedron Lett. 1970 967-970.

[8] T. Nagata, H. Matsubara, K. Kiyokawa, S. Minakata, Org. Lett. 2017, 19, 4672-4675.

[9] N. J. Foulger, B. J. Wakefield, Tetrahedron Lett. 1972, 4169-4170

[10] a) E. C. Ashby, L.-C. Chao, H. M. Neumann, J. Am. Chem. Soc. 1973 , 95, 5186-5191; b) E. C. Ashby, L.-C. Chao, H. M. Neumann, J. Am. Chem. Soc. 1973, 95, 4896-4904.

[11] D. R. Armstrong, W. Clegg, M. MacGregor, R. E. Mulvey, P. A. O'Neil, J. Chem. Soc., Chem. Commun. 1993, 608-610.

[12] a) J.-M. Mattalia, C. Marchi-Delapierre, H. Hazimeh, M. Chanon, ARKIVOC 2006, (iv), 90-118; b) J.-M. R. Mattalia, Beilstein J. Org. Chem. 2017, 13, 267-284.

[13] P. C. Too, G. H. Chan, Y. L. Tnay, H. Hirao, S. Chiba, Angew. Chem., Int. Ed. 2016, 55, 3719-3723.
[14] a) D. S. C. Black, J. E. Doyle, Aust. J. Chem. 1978, 31, 2323-2326; b) J.M. Mattalia, N. Bodineau, J.-C. Negrel, M. Chanon, J. Phys. Org. Chem. 2000, 13, 233-236

[15] S. S. Kulp, A. Romanelli, Org. Prep. Proced. Int. 1992, 24, 7-12.

[16] a) F. F. Blicke, E.-P. Tsao, J. Am. Chem. Soc. 1953, 75, 5587-5590; b) F. F. Blicke, E.-P. Tsao, J. Am. Chem. Soc. 1954, 76, 2203-2206; c) G. A. Brine, K. G. Boldt, D. Prakash, D. J. Kotchmar, V. C. Bondeson, D. J. Bradley, P. Singh, F. I. Carroll, J. Chem. Soc., Perkin Trans. 1 1991, 1809-1814; d) J. D. Hull, F. Scheinmann, N. J. Turner, Tetrahedron: Asymmetry 2003, 14, 567-576; e) E. M. Schultz, J. Am. Chem. Soc. 1952, 74, 5793-5794.

[17] J. A. Milligan, C. A. Busacca, C. H. Senanayake, P. Wipf, Org. Lett. 2016 , 18, 4300-4303.

[18] a) A. A. Fokin, T. S. Zhuk, S. Blomeyer, C. Perez, L. V. Chernish, A. E. Pashenko, J. Antony, Y. V. Vishnevskiy, R. J. F. Berger, S. Grimme, C. Logemann, M. Schnell, N. W. Mitzel, P. R. Schreiner, J. Am. Chem. Soc. 2017, 139, 16696-16707; b) S. Rösel, J. Becker, W. D. Allen, P. R. Schreiner, J. Am. Chem. Soc. 2018, 140, 14421-14432; c) P. R. Schreiner, L. V. Chernish, P. A. Gunchenko, E. Y. Tikhonchuk, H. Hausmann, M. Serafin, S. Schlecht, J. E. P. Dahl, R. M. K. Carlson, A. A. Fokin, Nature 2011, 477, 308-311; d) J. P. Wagner, P. R. Schreiner, Angew. Chem., Int. Ed. 2015, 54, 12274-12296.

[19] J. T. Reeves, C. A. Malapit, F. G. Buono, K. P. Sidhu, M. A. Marsini, C. A. Sader, K. R. Fandrick, C. A. Busacca, C. H. Senanayake, J. Am. Chem. Soc. 2015, 137, 9481-9488.

[20] C. A. Malapit, I. K. Luvaga, J. T. Reeves, I. Volchkov, C. A. Busacca, A. R. Howell, C. H. Senanayake, J. Org. Chem. 2017, 82, 4993-4997.

[21] TURBOMOLE V7.2 2017, a development of University of Karlsruhe and Forschungszentrum Karlsruhe GmbH, 1989-2007, TURBOMOLE GmbH, since 2007; available from http://www.turbomole.com.

[22] This difference has to be attributed to three factors: i) the B3LYP formulation is not the same in TURBOMOLE and in Gaussioan09; ii) our basis set is somewhat larger; iii) we do not employ here an implicit solvent model

[23] F. J. Weiberth, S. S. Hall, J. Org. Chem. 1987, 52, 3901-3904.

[24] In studies on rhodium catalyzed transnitrilation of aryl boronic acids with DMMN the mechanism proposed involves the fragmentation of a ketenimine intermediate efficiently promoted by a strong base $\left(\mathrm{Cs}_{2} \mathrm{CO}_{3}\right)$. Under mild basic conditions (proton sponge) this intermediate is stable and can be hydrolyzed into a ketone. See: C. A. Malapit, J. T. Reeves, C. A. Busacca, A. R. Howell, C. H. Senanayake, Angew. Chem., Int. Ed. 2016, 55, 326-330; C. A. Malapit, D. R. Caldwell, I. K. Luvaga, J. T. Reeves, I. Volchkov, N. C. Gonnella, Z. S. Han, C. A. Busacca, A. R. Howell, C. H. Senanayake, Angew. Chem., Int. Ed. 2017, 56, 6999-7002

[25] a) A. D. Becke, Phys. Rev. A 1988, 38, 3098-3100; b) A. D. Becke, J. Chem. Phys. 1993, 98, 5648-5652; c) P. A. M. Dirac, Proc. R. Soc. London, Ser. A 1929, 123, 714-733; d) C. Lee, W. Yang, R. G. Parr, Phys. Rev. B 1988, 37, 785-789; e) J. C. Slater, Phys. Rev. 1951, 81, 385-390; f) S. H. Vosko, L. Wilk, M. Nusair, Can. J. Phys. 1980, 58, 1200-1211

[26] a) Perdew, Wang, Phys. Rev. B 1992, 45, 13244-13249; b) J. P. Perdew, K. Burke, M. Ernzerhof, Phys. Rev. Lett. 1996, 77, 3865-3868; c) J. P. Perdew, M. Ernzerhof, K. Burke, J. Chem. Phys. 1996, 105, 9982-9985.

[27] S. Grimme, J. Antony, S. Ehrlich, H. Krieg, J. Chem. Phys. 2010, 132, 154104. 
[28] Y. Zhao, D. G. Truhlar, Theor. Chem. Acc. 2008, 120, 215-241.

[29] S. Grimme, J. Chem. Phys. 2003, 118, 9095-9102.

[30] a) C. Hattig, D. P. Tew, A. Kohn, J. Chem. Phys. 2010, 132, 231102; b) D. P. Tew, W. Klopper, C. Neiss, C. Haettig, Phys. Chem. Chem. Phys. 2007, 9, 1921-1930.

[31] a) F. Weigend, Phys. Chem. Chem. Phys. 2006, 8, 1057-1065; b) F. Weigend, R. Ahlrichs, Phys. Chem. Chem. Phys. 2005, 7, 3297-3305.

[32] F. A. Bischoff, S. Wolfsegger, D. P. Tew, W. Klopper, Mol. Phys. 2009, 107, 963-975.

[33] P. Nava, Y. Carissan, Phys. Chem. Chem. Phys. 2014, 16, 16196-16203.

[34] D. J. Cram, Fundamentals of Carbanion Chemistry, Academic Press, New-York, 1965, chapter 1.

[35] Studies suggest that the phenyl group has a larger size than methyl, see: M. Charton, J. Am. Chem. Soc. 1975, 97, 1552-1556; S. E. Boiadjiev, D. A. Lightner, J. Am. Chem. Soc. 2000, 122, 11328-11339; A. Y. Meyer, J. Chem. Soc., Perkin Trans. 21986, 1567-1572

[36] A. Holm, E. Huge-Jensen, Acta Chem. Scand., Ser. B 1974, 28, 705-710.

[37] For an estimation of electronegativity of the $\mathrm{MgBr}$ group, see: N. Inamoto, S. Masuda, Chem. Lett. 1982, 1003-1006; J. E. Huheey, J. Phys. Chem. 1965, 69, 3284-3289

[38] a) B. Gemünd, H. Nöth, H. Sachdev, M. Schmidt, Chem. Ber. 1996, 129, 1335-1344; b) K. Ruhlandt-Senge, J. J. Ellison, R. J. Wehmschulte, F. Pauer, P. P. Power, J. Am. Chem. Soc. 1993, 115, 11353-11357; c) M. Tacke, Eur. J. Inorg. Chem. 1998, 537-541; d) C. Üffing, R. Köppe, H. Schnöckel, Organometallics 1998, 17, 3512-3515.

[39] V. Dhindhwal, N. Sathyamurthy, J. Chem. Sci. 2016, 128, 1597-1606.

[40] a) E. Carl, D. Stalke, in Lithium Compounds in Organic Synthesis (Eds.: R. Luisi, V. Capriati), Wiley-VCH, Weinheim, 2014, pp. 3-31; b) V. H. Gessner, C. Däschlein, C. Strohmann, Chem. Eur. J. 2009, 15, 3320 3334; c) H. J. Reich, Chem. Rev. 2013, 113, 7130-7178.

[41] M. T. Rodgers, P. B. Armentrout, Chem. Rev. 2016, 116, 5642-5687.

[42] a) P. M. Bendale, B. R. Chowdhury, B. M. Khadilkar, Indian J. Chem. 2001, 40B, 433-435; b) C. E. Berkoff, D. E. Rivard, D. Kirkpatrick, J. L. Ives, Synth. Commun. 1980, 10, 939-945.

[43] G. B. Gregory, A. L. Johnson, W. C. Ripka, J. Org. Chem. 1990, 55, 1479 1483.

[44] a) M. Nambo, M. Yar, J. D. Smith, C. M. Crudden, Org. Lett. 2015, 17, 50-53; b) Y. N. Polivin, R. A. Karakhanov, T. S. Sheveleva, E. A. Ageev, A. S. Remizov, Bull. Acad. Sci. USSR, Div. Chem. Sci. 1991, 40, 25182520.

[45] a) D. Domon, M. Iwakura, K. Tanino, Tetrahedron Lett. 2017, 58, 19571960; b) S. Mylavarapu, M. Yadav, M. Bhanuchandra, Org. Biomol. Chem. 2018, 16, 7815-7819. 


\section{Entry for the Table of Contents}

Layout 1:

\section{FULL PAPER}

Imine adducts can undergo eliminations involving $\mathrm{C}-\mathrm{C}$ bond breakings. A computational study shows that such intermediates display stretched C-C bonds. Steric hindrance, carbanion stabilizing groups and leaving group-metal interactions favour this process.

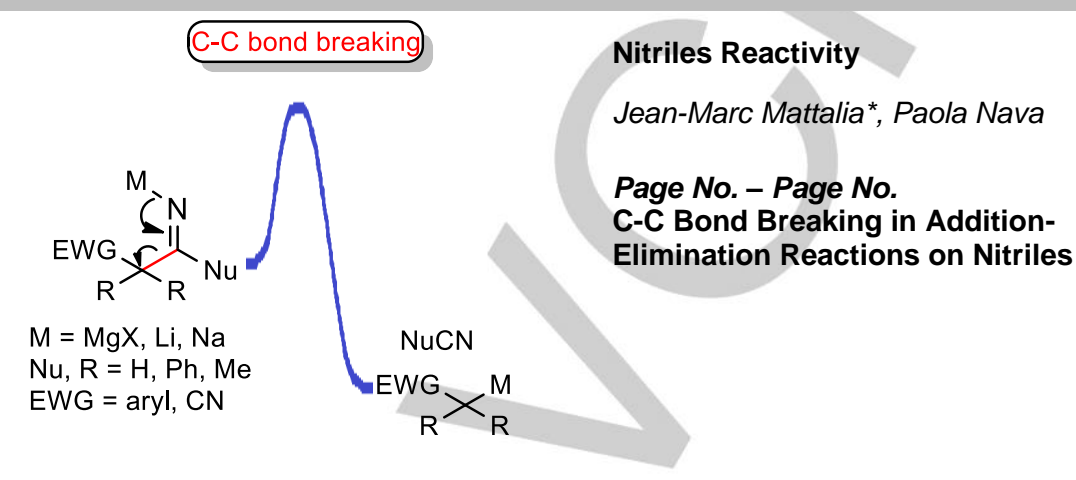

